ECCOMAS

Proceedia
M. Papadrakakis, M. Fragiadakis (eds.)

Rhodes Island, Greece, 15-17 June 2017

\title{
HYSTERETIC MODEL FOR THE EXPLICIT MATERIAL POINT METHOD
}

\author{
Christos D. Sofianos $^{1}$, Vlasis K. Koumousis ${ }^{1}$ \\ ${ }^{1}$ National Technical University of Athens \\ Institute of Structural Analysis \& Aseismic Research \\ Zografou Campus, 15780 Athens, Greece \\ e-mail: sofianoschristos@yahoo.gr, vkoum@central.ntua.gr
}

Keywords: Material Point Method, Hysteresis.

\begin{abstract}
The material point method (MPM) is an advancement of particle in cell method (PIC), in which Lagrangian bodies are discretized by a number of material points that hold all the properties and the state of the material ${ }^{[1]}$. All internal variables, stress, strain, velocity etc., that specify the current state and are required to advance the solution are stored in the material points. A background grid is employed to solve the governing equations by interpolating the material point data to the grid. The derived momentum conservation equations are solved at the grid nodes and information is transferred back to the material points and the background grid is reset. In this work the standard explicit MPM is extended to account for elastoplastic material behavior. The stress-strain constitutive law is determined according to the strain decomposition rule ${ }^{[2]}$ where the strain rates are uncoupled into an elastic and a plastic part. In order to account for the different phases during elastic loading or unloading and yielding two Heaviside type functions are introduced ${ }^{[3]}$. These act as switches and incorporate the yield function ${ }^{[8]}$. The final form of the constitutive stress-strain relation incorporates the tangent modulus of elasticity, which now includes the Heaviside functions and gathers all of the governing behavior, facilitating considerably the solution. Numerical results are presented that validate the proposed formulation in the context of the MPM and in comparison with Finite Element Method.
\end{abstract}

(C) 2017 The Authors. Published by Eccomas Proceedia.

Peer-review under responsibility of the organizing committee of COMPDYN 2017.

doi: $10.7712 / 120117.5686 .17699$ 


\section{INTRODUCTION}

The Material Point Method (MPM) is an extension of the Particle in Cell (PIC) ${ }^{[9]}$ method that was first developed for fluid mechanics problems. Many of the inherent numerical problems associated with PIC were alleviated in the fluid implicit particle method (FLIP) ${ }^{[10]}$. MPM was originally conceived as an extension of FLIP in solid mechanics. The most noticeable difference however, is that in MPM the description of motion and governing equations are derived from the weak form of the updated Lagrangian formulation.

The MPM utilizes both an Eulerian background grid and a Lagrangian discretization of the domain. It can be divided into two phases. First the material domain is represented by a set of particles (material points). All the information of the subdomain that each particle represents is hold in the material points (such as mass, momentum, velocity, strain or stresses etc.). This Lagrangian phase is followed by an Eulerian phase where a background grid is employed. Information is then transferred into the nodes of the grid where the governing equations are solved. After this convective phase the material point variables are updated and the background grid is reset to its original form. This means that the grid is not attached to the material points and does not follow the deformation. In addition, one of the advantages of the MPM is that the background grid can be arbitrarily chosen for convenience.

\section{MPM MODEL}

If $\Omega$ is the domain of the material, it can be represented by $N_{p}$ number of material points (or particles) as in Figure 1.

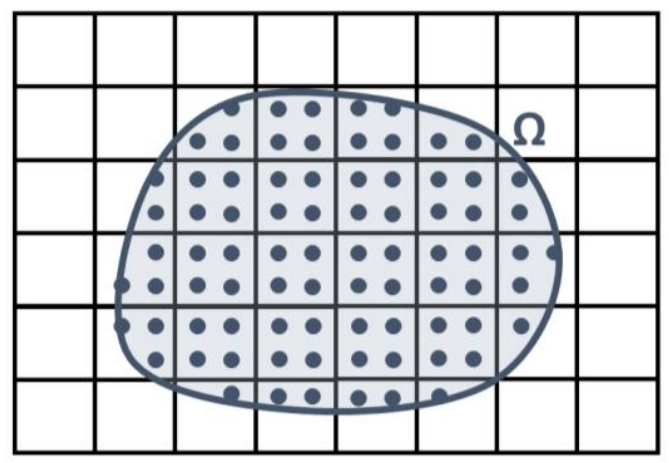

Figure 1: MPM discretization.

The material points are assigned a mass $m_{p}$, position $x_{p}$, velocity $v_{p}$ and stress $\sigma_{p}$. Since the total mass is divided into the material point set and the material points have constant mass through the simulation, the mass conservation is fulfilled. Conservation of momentum is:

$$
p \frac{d v}{d t}=\nabla \sigma+p b
$$

where $v$ is the velocity field, $\sigma$ is the Cauchy stress tensor, $p$ is the density and $b$ is the specific body force. In the previous relation $\nabla \sigma$ is the internal force while $p b$ is the external forces acting on the body. Moreover, if $E$ is the mechanical energy and $\varepsilon$ the strain tensor, the mechanical energy conservation equation is defined as:

$$
p \frac{d E}{d t}=\sigma: \frac{d E}{d t}+p v \cdot b
$$


The strain rate is expressed in terms of the gradient velocity as:

$$
\frac{d E}{d t}=\frac{1}{2}\left(\nabla v+(\nabla v)^{T}\right)
$$

and for the linear elastic case the constitutive equation relating stresses and stresses can be expressed using the elasticity tensor $D$ as:

$$
\frac{d \sigma}{d t}=D \frac{d \varepsilon}{d t}
$$

The plane stress elasticity matrix $D$ is defined as:

$$
D=\left[\begin{array}{ccc}
\frac{E}{\left(1-v^{2}\right)} & \frac{E v}{\left(1-v^{2}\right)} & 0 \\
\frac{E v}{\left(1-v^{2}\right)} & \frac{E}{\left(1-v^{2}\right)} & 0 \\
0 & 0 & \frac{E}{2(1+v)}
\end{array}\right]
$$

where $E$ is the Young's modulus and $v$ is the Poisson ratio.

In the Material Point Method each particle represents a sub-domain of the whole domain $\Omega$. Using the Dirac delta function the mass density can be expressed as a function of the material point masses and the material point positions as:

$$
p=\sum_{i=1}^{N_{p}} m_{p} \delta\left(x-x_{p}\right)
$$

Similar to the Finite Element method the material gradients are calculated on a background computational grid. Shape functions are used to approximate the solution and the velocity and acceleration of any material point are expressed in terms of the nodal accelerations of the grid as:

$$
v\left(x_{p}\right)=\sum_{i=1}^{N} v_{i} N_{i}
$$

and:

$$
\alpha\left(x_{p}\right)=\sum_{i=1}^{N} \frac{d v_{i}}{d t} N_{i}
$$

where in both relations the summation refers to the nodes of the element of the background grid that the material point resides in. In this work the shape functions used are cubic BSplines ${ }^{[4],[5]}$. They have been shown to reduce the quadrature errors and the grid crossing errors associated with discontinuous shape functions. Applying the Galerkin method and using the previous relations in the momentum equation integrals are replaced by sums over all the material points and the momentum equation now reads:

$$
\sum_{i=1}^{N} m_{i} \frac{d v_{i}}{d t}=F_{i}^{\mathrm{int}}+F_{i}^{e x t}
$$

The internal and the external forces are defined as: 


$$
F_{i}^{\mathrm{int}}=-\sum_{i=1}^{N_{p}} \frac{m_{p, i}}{p} \sigma_{p, i} \nabla N_{i}
$$

and:

$$
F_{i}^{e x t}=\tau_{i}+b_{i}
$$

where the subscript $p, i$ denotes the material point $i$.

Although many implementations are possible the main algorithm of the MPM is described below:

Map mass, momentum and internal forces from the particles to the background grid nodes:

$$
\begin{gathered}
m_{i}=\sum_{p=1}^{N_{p}} M_{p} N_{i} \\
(m v)_{i}=\sum_{p=1}^{N_{p}}(M v)_{p} N_{i} \\
F_{i}^{\mathrm{int}}=-\sum_{p=1}^{N_{p}} \frac{M_{p}}{p_{p}} \sigma_{p} \nabla N_{i}
\end{gathered}
$$

Compute the nodal force vector and apply the boundary conditions:

$$
F_{i}=F_{i}^{e x t}+F_{i}^{\mathrm{int}}
$$

Update nodal momenta at the nodes:

$$
(m v)_{i}=(m v)_{i}+F_{i} d t
$$

Update particle positions and velocities:

$$
\begin{gathered}
v\left(x_{p}\right)=v\left(x_{p}\right)+\left(\sum_{i=1}^{N} \frac{F_{i} N_{i}}{m_{i}}\right) d t \\
x_{p}=x_{p}+\left(\sum_{i=1}^{N} \frac{(m v)_{i} N_{i}}{m_{i}}\right) d t
\end{gathered}
$$

Employing the Modified Update Stress Last (MUSL) scheme the final step is to recalculate the grid nodal momentum based on the new particle velocities and from there calculate the particle strain and stress increments as:

$$
\begin{aligned}
& (m v)_{i}=\sum_{p=1}^{N_{p}} M_{p} v\left(x_{p}\right) N_{i} \\
& v_{i}=\frac{(m v)_{i}}{m_{i}} \\
& \Delta \varepsilon_{p}=\sum_{i=1}^{N} v\left(x_{p}\right) \nabla N_{i} d t \\
& \Delta \sigma_{p}=E \Delta \varepsilon_{p}
\end{aligned}
$$




\section{HYSTERETIC MODEL}

Based on the theory of classical plasticity the governing equations of plasticity are the flow rule, the yield condition, the consistency condition and the hardening rule. In this work kinematic hardening is considered. The plastic strain tensor $\left\{\dot{\varepsilon}^{p}\right\}$ can be expressed in terms of the flow rule $\Phi$ as:

$$
\left\{\dot{\varepsilon}^{p}\right\}=\dot{\lambda} \frac{\partial \Phi(\{\sigma\})}{\partial\{\sigma\}}
$$

where $\dot{\lambda}$ is the plastic multiplier and $\{\sigma\}$ is the stress tensor. The Kuhn-Tucker optimality conditions state that:

$$
\dot{\lambda} \geq 0 \quad \Phi \leq 0 \quad \dot{\lambda} \Phi=0
$$

Using the additive decomposition of the strain rates we derive:

$$
\{\dot{\varepsilon}\}=\left\{\dot{\varepsilon}^{e l}\right\}+\left\{\dot{\varepsilon}^{p l}\right\}
$$

The elastic part of the strain rates is connected to the stress rate tensor with the use of the elasticity tensor as:

$$
\{\dot{\sigma}\}=[D]\left\{\dot{\varepsilon}^{e l}\right\}
$$

Substituting equation (17) to (20) with the use of (19) we can write:

$$
\{\dot{\sigma}\}=[D]\left\{\{\dot{\varepsilon}\}-\dot{\lambda} \frac{\partial \Phi(\{\sigma\})}{\partial\{\sigma\}}\right\}
$$

Using the Kuhn-Tucker optimality condition in (18) and relation (21) and since at yield $\dot{\Phi}=0$ and $\dot{\lambda} \geq 0$ the following relation can be derived ${ }^{[8]}$ :

$$
\left(\frac{\partial \Phi(\{\sigma\})}{\partial\{\sigma\}}\right)^{T}\{\dot{\sigma}\}+\left(\frac{\partial \Phi(\{\sigma\})}{\partial\{\sigma\}}\right)^{T}\{\dot{\eta}\}=0
$$

Finally, the expression for the plastic multiplier becomes ${ }^{[8]}$ :

$$
\dot{\lambda}=\left(-C+\left(\frac{\partial \Phi(\{\sigma\})}{\partial\{\sigma\}}\right)^{T}[D] \frac{\partial \Phi(\{\sigma\})}{\partial\{\sigma\}}\right)^{-1}\left(\frac{\partial \Phi(\{\sigma\})}{\partial\{\sigma\}}\right)^{T}[D]\{\dot{\varepsilon}\}
$$

The previous equations hold when yielding has occurred. In order to smooth the transition from the elastic to the inelastic regime and generalize the plastic multiplier in the whole domain of the strain tensor as in ${ }^{[6]}$ and ${ }^{[7]}$, the following Heaviside functions are introduced:

$$
H_{1}=\left\{\begin{array}{ll}
1 & \Phi=0 \\
0 & \Phi<0
\end{array}, \quad H_{2}= \begin{cases}1 & \dot{\Phi}=0 \\
0 & \dot{\Phi}<0\end{cases}\right.
$$

This way a single expression is used for the plastic multiplier neglecting the need for a piece wise approach for the domains of the Kuhn-Tucker condition. 


$$
\dot{\lambda}=H_{1} H_{2}\left(-C+\left(\frac{\partial \Phi(\{\sigma\})}{\partial\{\sigma\}}\right)^{T}[D] \frac{\partial \Phi(\{\sigma\})}{\partial\{\sigma\}}\right)^{-1}\left(\frac{\partial \Phi(\{\sigma\})}{\partial\{\sigma\}}\right)^{T}[D]\{\dot{\varepsilon}\}
$$

The Heaviside functions are smoothed according to the following relations ${ }^{[3]}$ :

$$
H_{1}=\left|\frac{\Phi}{\Phi_{0}}\right|^{n}, \quad H_{2}=0.5+0.5 \operatorname{sign}\left(\{\varepsilon\}^{T}\{\dot{\sigma}\}\right)
$$

Finally substituting the plastic multiplier into equation (21) the following relation can be obtained relating stress and strain rates:

$$
\begin{gathered}
\{\dot{\sigma}\}=\left[E_{t}\right]\{\dot{\varepsilon}\} \\
{\left[E_{t}\right]=[D]\left([I]-\left|\frac{\Phi}{\Phi_{0}}\right|^{n}\left(0.5+0.5 \operatorname{sign}\left(\{\varepsilon\}^{T}\{\dot{\sigma}\}\right)\right)[R]\right)}
\end{gathered}
$$

This tangent elasticity modulus can now be incorporated in the MPM framework in relation (16) and the updated stresses can be calculated using the explicit scheme.

\section{NUMERICAL EXAMPLES}

\subsection{Cantilever beam}

In this example a cantilever beam subject to a point load on its free end is analyzed. The material is steel with $E=210 G P a, v=0.3$ and $s_{y}=240 M P a$. The beam has a length of $1 \mathrm{~m}$ and a height of $0.1 \mathrm{~m}$ while its width is $0.05 \mathrm{~m}$. The material point discretization is shown in Figure 2 . For the beam discretization 360 material points were used. The load is applied gradually over time in order to simulate a quasi-static conditions. The force-displacement diagram of the free end of the beam are presented in Figure 3 and are compared with results from Finite Element codes. Finally, the Von Mises stresses are presented in Figure 4. Results are in good agreement and the hysteretic MPM model can accurately predict both the displacement history of the beam as well as its stress state.

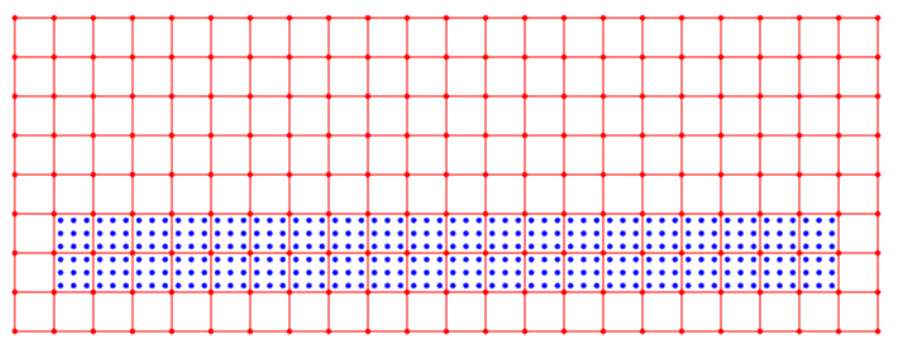

Figure 2: MPM discretization of cantilever beam with 360 material points. 


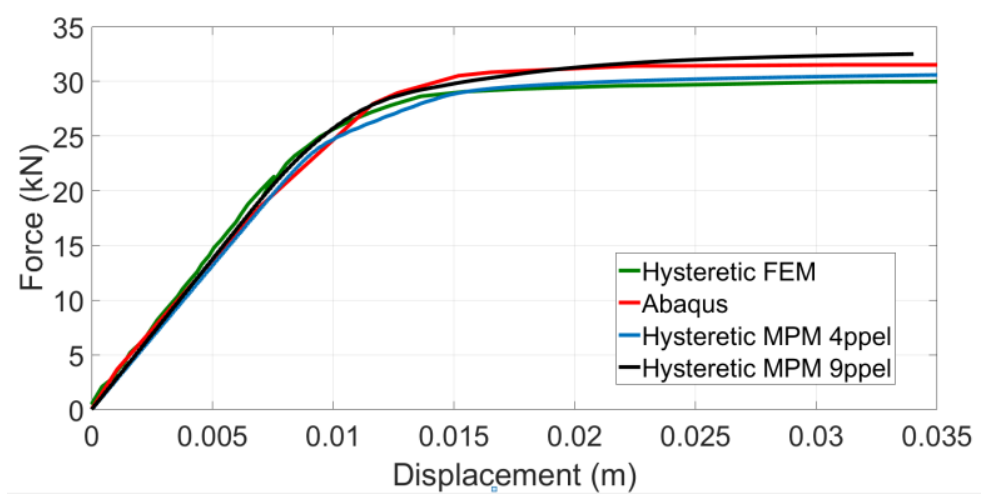

Figure 3: Force-displacement diagrams at the free end.

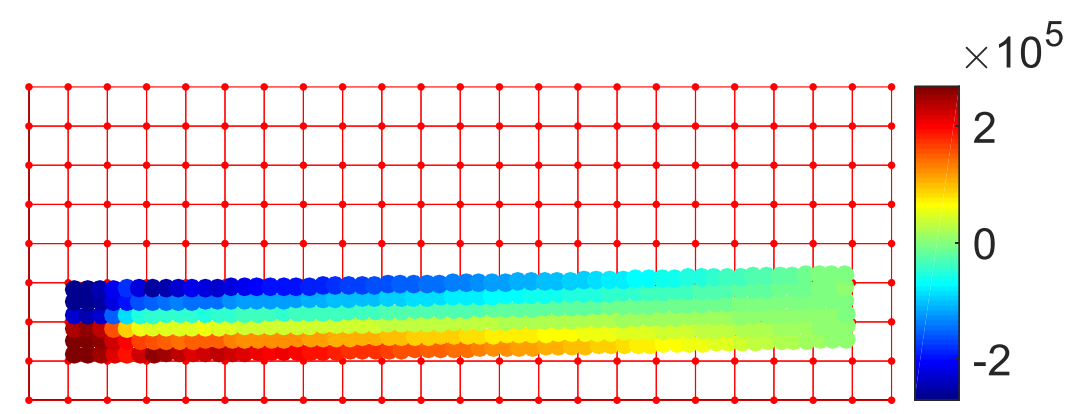

Figure 4: Von Mises stresses.

\subsection{Dynamic load cantilever beam}

For this second example another cantilever beam is considered with dimensions $1 \mathrm{x} 0.2 \mathrm{~m}$. The beam thickness is $0.02 \mathrm{~m}$. Material is steel S235 and 5\% hardening is considered. The beam is loaded at its free end with a sinusoidal load of $75 \mathrm{kN}$. In Figure 5 the history of the displacement of the free end of the beam is presented and compared with results from ANSYS Finite Element code. Plastic regions were developed in the support of the beam. Results are in good agreement verifying the proposed formulation in quasi-static and dynamic conditions.

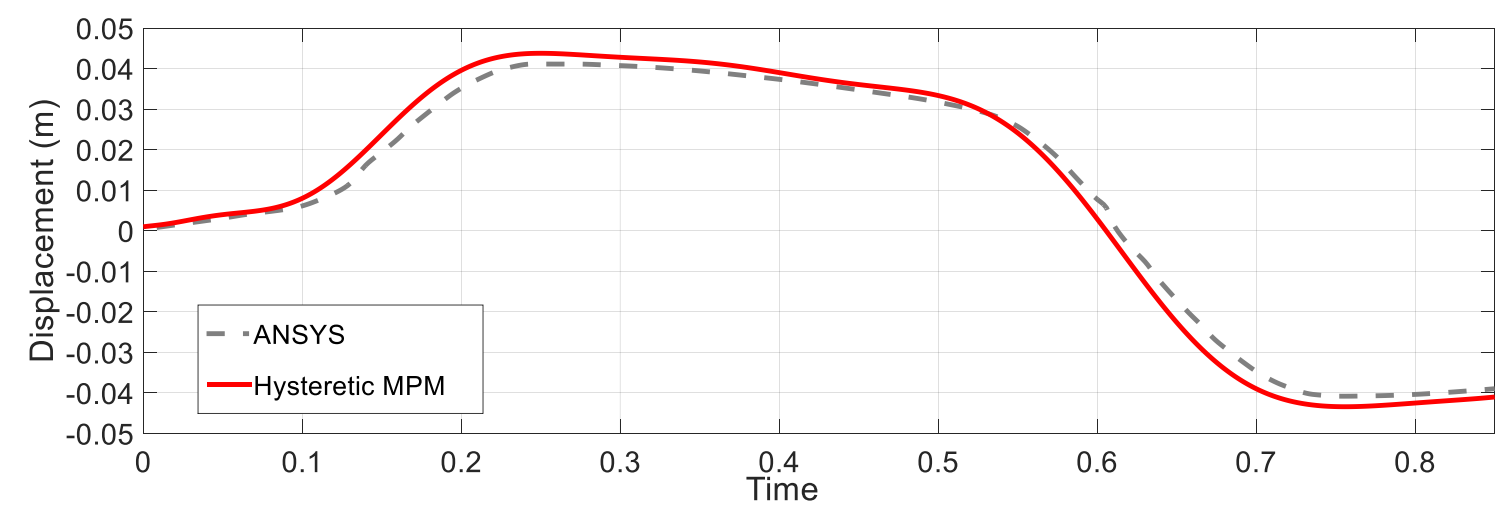

Figure 5: Free end displacement history.

\section{CONCLUSIONS}

- The Material Point Method is used in an explicit formulation to model dynamic or quasistatic loadings accounting for the inelastic behavior. 
- An hysteretic model has been presented for nonlinear analysis that accounts for smooth transition from the elastic to the inelastic regime.

- The hysteretic model has been efficiently incorporated into the MPM framework by modifying the tangent modulus of elasticity.

- High order cubic B-Splines have been used to minimize the grid crossing error and improve the accuracy of the MPM method.

- Numerical examples are compared with existing finite element codes and verify the proposed model ability to simulate hysteretic phenomena in the framework of MPM.

\section{ACKNOWLEDGMENTS}

The authors would like to acknowledge the support from the "RESEARCH PROJECTS FOR EXCELLENCE IKY/SIEMENS”.

\section{REFERENCES}

[1] D. Sulsky, Z. Chen and H. L. Schreyer, A particle method for history-dependent materials, Comput. Methods, Appl. Mech. Eng. 118,179 (1994).

[2] Lubliner J. Plasticity theory. New York. Dover Publications, Inc, 2008

[3] Bouc R. "Forced vibration of mechanical system with hysteresis", Proc. $4^{\text {th }}$ Conf. on Nonlinear Oscillation, Prague

[4] Andersen S., Andersen L., Analysis of spatial interpolation in the material-point method. Computers and Structures 88 (2010), 506-518.

[5] Steffen M, Kirby RM, Berzins M. Analysis and reduction of quadrature errors in the material point method (MPM). International Journal for Numerical Methods in Engineering;76:(2008), 922-48

[6] Triantafyllou S., Koumousis V., Bouc-Wen Hysteretic Plane Stress Element, Journal of Engineering Mechanics, 138 (2012), 235-246.

[7] Casciati F. (1995) Stochastic dynamics of hysteretic media. In: Krée P., Wedig W. (eds) Probabilistic Methods in Applied Physics. Lecture Notes in Physics, vol 451. Springer, Berlin, Heidelberg.

[8] Triantafyllou S.P., Koumousis V.K., An hysteretic quadrilateral plane stress element, Arch Appl Mech, 82 (2012), 1675-1687.

[9] Harlow F.H., The particle-in-cell computing method for fluid dynamics, Methods for Computational Physics, 3 (1964), 319-43.

[10] Brackbill J.U., Ruppel H.M., FLIP: A method for adaptivelyzoned,particle-incellcalculations of fluid flows in two dimensions. Journal of Computational Physics, 65(2), (1986), 314-343. 